Intervenção em Saúde do Trabalhador

\author{
Márcia da Silva Anunciação Lazarino ${ }^{a}$ \\ (iD) http://orcid.org/0000-0002-6458-2180 \\ Thais Lacerda e Silva ${ }^{b}$ \\ (D) https://orcid.org/0000-0001-9869-9654 \\ Elizabeth Costa Diasc \\ (iD) https://orcid.org/0000-0003-1708-1014
}

a Secretaria Municipal de Saúde de Betim, Centro de Referência em Saúde do Trabalhador. Betim, MG, Brasil.

b Escola de Saúde Pública de Minas Gerais, Superintendência de Educação e Trabalho em Saúde. Belo Horizonte, MG, Brasil.

' Universidade Federal de Minas Gerais (UFMG), Faculdade de Medicina, Departamento de Medicina Preventiva e Social. Belo Horizonte, MG, Brasil.

Contato:

Márcia da Silva Anunciação Lazarino

E-mail:

mslazarino@gmail.com

Trabalho baseado na dissertação de mestrado de Márcia da Silva Anunciação Lazarino, intitulada Apoio técnico e pedagógico para o desenvolvimento de ações de saúde do trabalhador na atenção básica: $a$ experiência do Centro de Referência em Saúde do Trabalhador de Betim, pela Universidade Federal de Minas Gerais, Belo Horizonte, 2017.

As autoras declaram que o estudo não foi subvencionado e que não há conflitos de interesses.

As autoras informam que o trabalho não foi apresentado em eventos científicos.

\section{Apoio matricial como estratégia para o fortalecimento da saúde do trabalhador na atenção básica}

\author{
Matrix support as a strategy to strengthen workers' health in \\ primary care
}

\section{Resumo}

Introdução: a garantia da atenção integral à saúde do trabalhador requer a incorporação sistemática de ações na atenção básica, sendo o apoio matricial uma estratégia potente para sua viabilização. Objetivo: descrever e analisar as práticas de apoio matricial desenvolvidas por um Centro de Referência em Saúde do Trabalhador (Cerest) junto à rede de serviços de atenção básica, com vistas à incorporação de ações de saúde do trabalhador. Métodos: estudo de natureza descritiva. Realizou-se entrevista semiestruturada com 41 profissionais que participaram do processo de matriciamento. Os dados foram analisados segundo a técnica de análise de conteúdo. Resultados: o apoio matricial possibilitou a ampliação de práticas que reconhecem o usuário enquanto trabalhador e o trabalho como determinante do processo saúdedoença. Com o aumento da resolutividade dos casos e maior articulação entre os profissionais do Cerest e da rede de atenção básica, houve redução dos encaminhamentos aos Cerest. Os desafios identificados incluem a necessidade de qualificar apoiadores e o enfrentamento da sobrecarga de trabalho e da ênfase nas ações assistenciais que ocorrem em detrimento das de vigilância, dificultando a integralidade do cuidado. Conclusão: o apoio matricial pode operar como estratégia potente de fortalecimento da saúde do trabalhador no Sistema Único de Saúde (SUS).

Palavras-chave: saúde do trabalhador; atenção primária à saúde; apoio matricial.

\begin{abstract}
Introduction: ensuring comprehensive care for workers' health requires systematic actions incorporation in primary health care, and including matrix support is a powerful strategy for its viability. Objective: to describe and analyze the matrix support practices adopted by a Occupational Health Reference Center (Cerest) for the incorporation of occupational health actions in the primary care network. Methods: a descriptive study was conducted using a semistructured interview with 41 health professionals who participated in the matrix process. The data were analyzed according to the technique of content analysis. Results: matrix support allowed the expansion of practices that recognize the primary health network user as a worker and the work as determinant of the healthdisease process. Increasing the resolution of cases and the greater articulation between the Cerest professionals and those of the primary care network made possible the referrals reduction to Cerest. Challenges identified include training of supporters, health professionals workload reduction, and greater emphasis on surveillance actions. Conclusion: matrix support can operate as a powerful strategy to strengthen occupational health in the Brazilian Unified Health System (SUS).
\end{abstract}

Keywords: occupational health; primary health care; matrix support. 


\section{Introdução}

O cuidado qualificado à saúde dos trabalhadores pelas equipes da Atenção Básica (AB) do Sistema Único de Saúde (SUS), incorporando sua inserção nos processos produtivos, tem sido considerado estratégico para a ampliação das ações de Saúde do Trabalhador (ST), na perpesctiva da integralidade, no contexto de crescimento do trabalho informal e precarizado no país ${ }^{1}$.

O tema tem recebido atenção de agências estrangeiras e de estudos de relevância nacional e internacional, os quais destacam o papel das equipes da $\mathrm{AB}$, tanto no sentido de desvelar o adoecimento relacionado ao trabalho quanto de promover o cuidado dos trabalhadores inseridos no setor informal, no trabalho precarizado e dos desempregados, em especial no atual cenário decorrente da reestruturação produtiva ${ }^{2,3}$. Particularmente nas situações de trabalho em domicílio, a $\mathrm{AB}$ é o único recurso entre as políticas públicas capaz de dar visibilidade a essa realidade, intervir sobre as más condições de trabalho e proteger a saúde desses trabalhadores ${ }^{4}$.

Desde a criação do SUS, distintas estratégias têm sido adotadas no processo de construção das ações de ST na rede de serviços, em cumprimento ao preceito constitucional regulamentado pela Lei 8.080 de 19 de setembro de 1990. A criação da Rede Nacional de Atenção Integral a Saúde do Trabalhador (Renast), em 2002, e a instituição da Política Nacional de Saúde do Trabalhador e da Trabalhadora (PNSTT), em 2012, são considerados marcos importantes desse processo ${ }^{5,6}$. De acordo com a PNSTT, toda rede de saúde deve estar comprometida com o desenvolvimento da atenção integral à ST, cabendo aos Centros de Referência em Saúde do Trabalhador (Cerest) prover apoio matricial para o desenvolvimento das ações de ST no âmbito da Atenção Básica de Saúde (ABS), nos serviços especializados de urgência e de emergência, bem como na promoção e vigilância à saúde ${ }^{6}$.

Entretanto, apesar das prescrições e normas existentes e do reconhecimento do papel do trabalho na determinação do processo saúde-doença dos trabalhadores, pelos profissionais das equipes da Estratégia de Saúde da Família (ESF), essas ações ainda são incipientes e/ou vulneráveis às conjunturas políticas ${ }^{7}$. Entre as dificuldades apontadas para a implementação da atenção à saúde dos trabalhadores na $\mathrm{AB}$ destacam-se: a verticalidade e centralização do planejamento da atuação das equipes; o predomínio de atividades assistenciais em detrimento das de vigilância e promoção da saúde; e a falta de suporte institucional, técnico e pedagógico especializado às equipes ${ }^{8}$.
O apoio matricial (AM) é um arranjo organizacional e uma metodologia de compartilhamento de saberes que viabiliza o suporte técnico pedagógico, e institucional, assegurando retaguarda especializada para as equipes responsáveis pelo desenvolvimento de ações para a população sob seu cuidado, denominadas Equipe de Referência (ER). Tem como objetivo ampliar o campo de atuação e qualificar as ações dos profissionais dessas equipes, contribuindo para o aumento da capacidade resolutiva das mesmas. Esta mudança nos modos de operar o cuidado em saúde possibilita fortalecer uma relação terapêutica longitudinal e o acompanhamento das condições de saúde de cada usuário, fazendo com que a equipe, responsável diretamente pelo cuidado, conheça na particularidade a história de vida de cada um, colocando-se como corresponsável pelo cuidado e outras ações que se desdobram a partir do encontro com o usuário. O cerne desse arranjo consiste na produção do cuidado por meio de um trabalho interdisciplinar, em relações horizontais de corresponsabilização sanitária entre apoiadores ou matriciadores e a equipe de referência ${ }^{9,10}$.

Experiências de AM em diferentes áreas têm demonstrado potencialidades e também dificuldades quanto ao uso da ferramenta para o cuidado em saúde. Na saúde mental (SM), o matriciamento possibilitou ampliação de acesso dos usuários aos serviços de saúde e o desenvolvimento de práticas mais consoantes aos principios da integralidade ${ }^{11,12} . \mathrm{Na}$ saúde do idoso, o estudo de Madureira e Bissoli ${ }^{13}$ destaca que no município de Vitória, o AM possibilitou a reorganização da atenção à saúde do idoso, bem como o acolhimento e a qualificação das demandas dos profissionais das ESF na gestão do cuidado da pessoa idosa, por meio de retaguarda assistencial e suporte técnico pedagógico pelos profissionais do Centro de Referência de Atendimento ao Idoso às ER. Estudo desenvolvido por Fonseca Sobrinho e colaboradores ${ }^{14}$, com vistas a estabelecer uma visão panorâmica da situação do AM no Brasil - por meio da análise de dados de avaliação externa do Programa Nacional de Melhoria do Acesso e da Qualidade da Atenção Básica (PMAQ-AB)- evidenciou que as atividades de $A M$ na $A B S$ são significativas, embora distribuídas desigualmente no território nacional, e contribuem para a melhoria da qualidade e do acesso da população à atenção à saúde no país.

Na ST, a literatura mostra experiências similares realizadas por Cerest. Destaca-se a de Amparo/SP, que revela mudança no olhar dos profissionais das ESF em relação aos trabalhadores, às relações trabalho-saúde no cuidado e às possibilidades de superar a abordagem tradicional de ações médico assistenciais individuais para ações coletivas de prevenção, promoção e vigilância. A qualidade do vínculo 
entre os profissionais da ABS e do Cerest tem sido apontada como importante desdobramento, resultando em maior responsabilização sobre a saúde dos trabalhadores ${ }^{15}$.

Assim, diante da necessidade de incorporação sistemática das ações de ST na ABS e das potencialidades inerentes à estratégia do $\mathrm{AM}$, este estudo descreve e analisa uma experiência de intervenção na $\mathrm{AB}$, ativada e desenvolvida por um Cerest regional, com a finalidadede de contribuir para a inserção da ST no cotidiano de trabalho das ESF.

\section{Métodos}

Trata-se de estudo de natureza descritiva a respeito de experiência de AM desenvolvida por um Cerest regional localizado no município de Betim no estado de Minas Gerais, junto à rede de serviços de AB do município.

A coleta de dados foi realizada por meio de entrevistas semiestruturadas feitas individualmente com diferentes profissionais envolvidos na implementação do AM em ST, no Cerest e na AB. O critério de inclusão dos participantes do Cerest foi: ser ou ter sido matriciador em ST de ESF e estar trabalhando no período da entrevista, entre 26/12/2016 a 13/01/2017. O grupo de entrevistados da AB foi formado por profissionais em exercício na Unidade Básica de Saúde (UBS) matriciada há mais de seis meses. Foram excluídos aqueles que se recusaram a participar ou que estavam ausentes do serviço no período de realização das entrevistas, ou seja, de 08/02 a 04/03/2017. Identificados aqueles que preenchiam os critérios de inclusão, procedeu-se o sorteio aleatório dos que seriam entrevistados.

$\mathrm{O}$ roteiro das entrevistas buscou estimular os participantes a emitirem suas opiniões, sentimentos e significados sobre a experiência vivida ${ }^{16,17}$. As entrevistas foram gravadas e posteriormente transcritas. Os entrevistados foram identificados com códigos: profissionais do Cerest pela letra " $\mathrm{C}$ ", seguida dos números de 1 a 8 de acordo com a ordem da transcrição das gravações. Para a identificação dos profissionais da AB foi definido: "A" para os agentes comunitários de saúde (ACS); "E” para enfermeiros e "M" para médicos, seguida dos números de 1 a 11, de acordo com a ordem da transcrição das gravações. Os entrevistados foram previamente esclarecidos sobre a pesquisa e assinaram o Termo de Consentimento Livre e Esclarecido (TCLE). O projeto foi aprovado pelo Comitê de Ética e Pesquisa da Universidade Federal de Minas Gerais sob o número ETIC 459/08.

Os dados obtidos foram organizados com a utilização de planilha eletrônica Microsoft Excel e analisados segundo a técnica de análise de conteúdo proposta por Bardin ${ }^{18}$, buscando identificar as categorias conforme temas que emergiram do texto. Assim, procedeu-se inicialmente uma pré-análise do material, realizada por meio de uma leitura flutuante, seguida de leitura exploratória para identificação dos núcleos de sentido, considerando as regularidades do discurso e os sentidos frequentes e ímpares presentes nas falas. Por fim, realizou-se a categorização, reagrupando as informações obtidas, considerando as seguintes categorias: a) reorientação de práticas de atenção à saúde dos trabalhadores; b) espaço de ensino-aprendizagem e c) limites e desafios do matriciamento em ST.

\section{Resultados e discussão}

Foram entrevistados 41 profissionais: 8 do Cerest, que atuavam como apoiadores, e 33 profissionais de ESF (ER) de 11 UBS onde o processo de matriciamento foi desenvolvido. Em cada UBS participaram um médico, um enfermeiro e um ACS. A seguir, são apresentadas as percepções desses profissionais em relação ao AM, buscando conhecer e discutir os modos de operar a ferramenta, suas concepções, resultados, potencialidades e dificuldades.

\section{Reorientação de práticas de atenção à saúde dos trabalhadores}

A reorientação de práticas dos profissionais das ESF, a partir do matriciamento, aparece evidente nas narrativas dos entrevistados. Os participantes foram unânimes em considerar que o matriciamento possibilitou intervenções mais resolutivas, qualificando o cuidado dos trabalhadores na ABS, por meio do trabalho compartilhado, conforme explicitado na fala de M4:

\footnotetext{
Foi resolutivo em muitos casos que o paciente ou ia ficar sem solução ou ia demorar anos para conseguir um posicionamento do médico especialista. (M4)
}

Observa-se nesse relato que o contato direto entre o médico da $\mathrm{AB}$ e o especialista de apoio em ST foi determinante para a resolução dos casos. Este é um dos propósitos básicos do AM, conforme salientam Campos e Domitti ${ }^{19}$ : "o apoio matricial busca personalizar os sistemas de referência e contra-referência [...]. A decisão sobre o acesso de um caso a um apoio especializado, seria, em última instância, tomada de maneira interativa, entre o profissional de referência e apoiador" (p. 401).

O encontro entre as equipes de referência e apoiadores, conforme destaca Campos ${ }^{9}$, pressupõe o trabalho compartilhado e a corresponsabilidade dos envolvidos com a produção de saúde, 
ampliando e qualificando o campo de atuação das equipes de referência.

O matriciamento garante que um profissional esteja mais perto do outro profissional, então que você consiga uma troca de informação, uma discussão de caso, da atenção básica entrar em contato, com o técnico e discutir diretamente com ele, de criar uma referência mesmo em saúde do trabalhador [...]. O matriciamento oferece essa possibilidade, que as outras capacitações às vezes não dá. (C1)

Para os profissionais das ESF, o matriciamento possibilitou a ampliação do olhar sobre o trabalho enquanto determinante do processo saúde-doença, preocupação evidenciada, por exemplo, em relação ao estabelecimento entre a queixa/doença referida pelo usuário e o trabalho.

Alguns casos que às vezes você nem via como doença ocupacional que você passa a ver, né, que você enxerga bem nessa questão aí. (M8)

Eu peguei um caso que era uma dermatite que eu até discuti com o Cerest, a gente, foi investigando produtos pra tentar identificar, e descobriu-se que o estresse do trabalho que tava provocando, e era na pele, né, e hoje em dia ela saiu do emprego e tá bem. Mas foi detectado isso aí, um caso interessante que foi trabaIhado junto com o Cerest. (M11)

A gente observa as queixas do usuário, né, então se ele, vamos supor, tá queixando muita dor na coluna, a gente procura saber se não é o trabalho que tá acarretando isso. (A1)

Também foi destacado por um dos profissionais entrevistados que o processo de AM contribuiu para ampliar o conhecimento sobre o trabalho desenvolvido na $\mathrm{AB}$ :

O matriciamento é uma possibilidade do pessoal de saúde do trabalhador entender mais o nosso trabaIho, e vice versa. (E8)

Conforme salientam Dias e Lacerda-Silva ${ }^{20}$, um pré-requisito para o desenvolvimento do matriciamento é o conhecimento sobre o processo de trabalho das equipes e as principais demandas e problemas de saúde que envolvem a relação trabalho/saúde/doença das pesssoas que vivem nos territórios sob responsabilidade sanitária das ESF.

Na experiência de AM em SM, Jorge et al. ${ }^{21}$ destacam a possibilidade de troca de conhecimentos entre os diversos núcleos de saberes, apontando para uma ação dialogada, interdisciplinar e de clínica ampliada. Essas mudanças aproximam-se da afirmação de Campos ${ }^{9}$ sobre o AM como dispositivo potente para alterar a lógica de cuidado focado apenas no indivíduo. O matriciamento assume, como caráter essencial, a interdisciplinaridade das ações, característica evidenciada nos discursos dos profissionais tanto do Cerest quanto das ESF, que o percebem como estratégia facilitadora do diálogo e da troca de saberes entre as várias categorias profissionais.

\section{Espaço de ensino-aprendizagem}

O matriciamento em ST foi considerado pelos entrevistados como oportunidade para adquirir novos conhecimentos ou retrabalhar concepções e conceitos prévios.

\begin{abstract}
Eu adquiri muitos conhecimentos que eu não tinha, que o matriciamento trouxe, né, de experiências de diferentes profissionais e coisas novas que eu não sabia: do direito do trabalhador, do quê que a gente servidor pode fazer frente a um processo que vem de fora, que são dos usuários, eu não, a gente não tinha. (E4)
\end{abstract}

A ausência e/ou pouca abordagem de conceitos e ferramentas do campo da ST nos cursos de graduação em saúde foi apontado por alguns dos entrevistados, que reconheceram o matriciamento como uma possibilidade de aprender questões que não tinham sido discutidas na graduação e suprir essa lacuna.

Em relação aos apoiadores, o matriciamento foi reconhecido como uma possibilidade de conhecerem parte da realidade vivida pelos profissionais da ABS: Eu pude conhecer melhor a outra ponta, a atenção
básica, como que é a rotina, pude estar mais próxima
dessa vivência, do dia a dia deles. (C3)

Eles também passam muita coisa pra gente que às vezes a gente nem tem ideia; então assim, é uma troca de experiências. (C8)

Estas falas apontam e reforçam para a troca de experiências como sendo um caminho potente para a construção e reconstrução de conhecimento.

Observa-se que o conhecimento sobre o processo de trabalho na ABS foi considerado importante para o desenvolvimento de ações compartilhadas entre as equipes. Esta percepção está em consonância com o fundamento da Política Nacional de Educação Permanente (PNEP), de que os processos formativos devem considerar e ter como ponto de partida os processos de trabalho e as necessidades de saúde dos indivíduos e coletividades.

Outra concepção sobre o AM, como um espaço de ensino e de aprendizagem, pode ser identificada na fala de um dos apoiadores:

Conseguimos levar algum conhecimento pra eles? Claro que a gente leva, claro que eles absorvem muito isso. (C5) 
Embora esta fala aponte e reconheça os distintos saberes existentes entre profissionais que atuam na $A B$ e no serviço especializado, também convida a pensar sobre as concepções de ensino e de aprendizagem que permeiam as práticas dos apoiadores. A expressão "levar conhecimento" sugere uma concepção de ensino e aprendizagem unilateral e hierárquica, ao contrário da proposta de educação dialógica e simétrica, que deve orientar os encontros do matriciamento.

Na mesma linha, Jorge et al. ${ }^{21}$, em estudo sobre o AM em SM, chamam atenção para as evidências de que a operacionalização do matriciamento vem se configurando mais como transferência de saber especializado do que propriamente troca de saberes entre profissionais que atuam nos diferentes níveis de atenção. Nas palavras dos autores: "a ênfase técnico-pedagógica do matriciamento evidencia a possível unilateralidade que pode cristalizar a Atenção Básica apenas no lugar de aprendiz, quando seu potencial está justamente na proximidade com a vida familiar e comunitária dos usuários" (p. 70). Neste sentido é preciso desenvolver iniciativas de discussão sobre o papel dos apoiadores, de modo que possam ressignificar sua atuação.

Ainda nesta mesma linha, alguns apoiadores referiram que não se sentem preparados para o exercício desta função. Grande parte dos profissionais do Cerest é formada em graduações que normalmente desenvolvem a assistência de forma individual:

Na minha formação eu nunca tive, é, essa experiência assim, a gente forma muito voltado pra atendimento da população. (C5)

Como é uma coisa que eu não tinha formação [...] Como fazer? (C1)

Estas percepções apontam para uma valorização das instituições formadoras do núcleo de conhecimento de cada profissão, não preparando o profissional para o trabalho interdisciplinar proposto pelo matriciamento. Embora os matriciadores façam parte de um serviço especializado em ST, cada profissional tem a sua especialidade. É no cotidiano de trabalho que se formam, de fato, no campo da ST.

Assim, é importante estar atento para o fato de que ser especialista em ST não torna o profissional automaticamente um apoiador de profissionais da ABS para o cuidado aos usuários trabalhadores. A função de apoiador exige um processo de formação, conforme explicita um dos entrevistados:

Eu acho que precisa de um suporte pra nós, profissionais que estamos no matriciamento, ter mais respaldo pra gente, pras nossas reuniões acontecerem, continuar nas nossas capacitações da gente também, porque como é algo novo, né? (C6)
A pouca qualificação para a função de apoiador contribui para o sentimento de insegurança expresso por muitos dos entrevistados:

Ir para a reunião com as ESF sem saber quais casos serão levados para o debate gera ansiedade e insegurança em alguns membros da equipe. (C2)

Este fato faz com que os técnicos queiram ser acompanhados pelos colegas de equipe que eles consideram mais habilitados:

[...] quando você vai com outro, outro membro da equipe porque as discussões ficam melhores. (C6)

Ter dois profissionais, facilita. (C2)

Esta sensação de insegurança influencia na escolha das metodologias de apoio selecionadas pelos apoiadores que, algumas vezes, não estimulam a discussão de casos e propõem encontros em torno do debate de temas específicos e previamente determinados:

Trabalhar tema específico também facilita, porque você consegue fazer um direcionamento, é, melhor. (C2)

Em meio a esta discussão, é importante retomar a intencionalidade central do AM de ampliar a capacidade de análise das equipes de referência para lidar com seus casos e, por sua vez, ampliar a capacidade resolutiva $^{22}$. Esta noção provoca a reflexão sobre o fato de que ser especialista não significa ter resposta para a resolução imediata do caso. A construção das intervenções para os problemas e necessidades de saúde apresentadas deve vir a partir da troca de saberes entre apoiadores e profissionais de referência. Neste sentido, o matriciamento requer um trabalho interdisciplinar e encontros sistemáticos e de estudo entre as duas equipes.

Assim, considerando que a prática do AM pressupõe uma dimensão de apoio pedagógico e de suporte assistencial especializado, realizado com as equipes de referência, sua operacionalização requer a qualificação específica dos profissionais do Cerest.

No entanto, mesmo frente às diferenças de compreensões sobre o AM identificadas nas entrevistas e das dificuldades relacionadas à preparação dos profissionais do Cerest para realização do apoio, observa-se que o matriciamento em ST se mostrou potente para a mudança de práticas de cuidado à saúde e para a incorporação do trabalho como determinante do processo saúde-doença, no cotidiano de trabalho das equipes de SF.

Tinha ações que eu não fazia e algumas que eu nem me lembrava... E outra coisa [...] facilitou o contato. Hoje em dia o pessoal do Cerest conhece a gente. Então dá pra discutir um caso ainda até mais fácil. (M11) 
Quando começou o matriciamento, que tem dois anos, né, abriu o nosso leque, então a gente começou a perceber mais esses usuários, pensar mais sobre isso, que ás vezes eles viam com uma queixa que tava relacionada ao trabalho e a gente não enxergava, então abriu a nossa percepção, a gente ficou mais alerta a esse tipo de queixa, associar mais ao trabalho, que tinha ficado meio despercebido, eu acho. (E4)

Neste sentido, o matriciamento em ST tem operado como uma estratégia de educação permanente em saúde, possibilitando mudanças nas práticas de saúde desenvolvidas e também na própria forma de organização do trabalho.

\section{Limites e desafios do matriciamento em saúde do trabalhador}

Entre as dificuldades apontadas pelos profissionais das ESF para participar das atividades de AM em ST, estão as exigências relativas aos procedimentos clínicos habituais da $\mathrm{AB}$ e a pressão para o atendimento às demandas estabelecidas pelos programas do Ministério da Saúde:

Outra coisa que pode atrapalhar é a grande demanda do serviço, por exemplo, quando a gente tá em reuniões ou capacitações dentro da unidade, chega muita coisa pra gente, o tempo todo a gente é interrompido. (M11)

Na mesma linha, a ênfase nas ações assistenciais, em detrimento das de vigilância e promoção da saúde, dificulta o desenvolvimento do cuidado integral em ST. Embora a AB seja crescentemente reconhecida como um lócus privilegiado para a incorporação de ações de vigilância de ambientes de trabalho domiciliar ${ }^{20}$, as falas dos profissionais expressam essa dificuldade:

A gente não pode sair pra ver condição de trabalho de ninguém aqui, entendeu. O foco aqui é outro. (M8)

[...] vigilância in loco, não, no local de trabalho, não. (E5)

A gente ainda não fez essa de visitar o lugar de serviço, né. (E4)

No âmbito das ações de vigilância em ST, as entrevistas evidenciaram a realização de identificação, notificação e análise dos agravos à saúde relacionados ao trabalho:

[...] vigilância só da epidemiológica mesmo, né, que seria as fichas de notificação, né, que a gente muitas vezes usa pra notificar algum agravo. (E10)

A gente faz notificação. (E3)

Entretanto, observou-se que a identificação de situações de risco e vulnerabilidades decorrentes dos processos produtivos existentes no território é incipiente e, quando identificada a necessidade de vigilância de ambientes e condições de trabalho, os profissionais fazem contato com a equipe do Cerest:

[...] agora, vigilância de ambiente, nós já tivemos problema e aí eu solicitei pro Cerest. (M4)

É interessante destacar que a lógica de cuidado assistencialista também marca a atuação do Cerest, sendo ressaltada por um dos profissionais a necessidade de mudanças, especialmente diante da necessidade de fortalecer o AM:

Nós somos muito voltados pra assistência, e aí a gente não consegue fazer tantas ações de prevenção e de matriciamento. (C4)

A rotatividade dos médicos da $\mathrm{AB}$ e o longo período demandado para a reposição do profissional foram apontados como dificuldades no processo de matriciamento em ST. Em que pese todos os esforços empreendidos pelo Ministério da Saúde (MS) para a fixação de médicos na Saúde da Família, a rotatividade ainda é significativa, conforme expressa um dos profissionais do Cerest:

A alta rotatividade dos profissionais é um grande problema que faz com que você tá sempre recomeçando. (C2)

Outras dificuldades mencionadas foram: a incompatibilidade de horários observada entre as equipes dos dois serviços; o cancelamento de reuniões e a necessidade de saída de profissionais durante as discussões, para atender outras demandas, em geral, de assistência. Em relação às dificuldades específicas das equipes do Cerest, foram mencionadas: insegurança e falta de qualificação do apoiador; falta de carro para realização do deslocamento dos apoiadores para as reuniões nas UBS; o fato de ter que realizar, muitas vezes, a atividade de apoio por apenas um profissional do Cerest; entre outras. A realização do apoio às ER, em dupla, e de modo especial, com a presença de um colega mais experiente foi apontado por profissionais como sendo importante para o desenvolvimento das ações.

A carga de trabalho excessiva resultante da demanda sobre os profissionais de ambas as equipes, e de modo particular, sobre as ESF; a alta rotatividade de profissionais e a dificuldade de compatibilizar as agendas entre os serviços também foram ressaltadas por Santos e Lacaz ${ }^{15}$, no estudo sobre a experiência de AM em ST no município de Amparo/SP.

Porém, apesar das dificuldades identificadas, a equipe do Cerest considera importante ampliar o matriciamento para todas as UBS, conforme expresso na fala: 
Ah, eu queria que fosse em todas [...] [pausa] É... Isso me emociona porquê eu acho muito importante sabe. (C1)

\section{Considerações finais}

A AB se apresenta crescentemente como importante estratégia para a atenção à saúde dos trabalhadores no SUS, especialmente pela capilaridade da rede de serviços, que possibilita levar o cuidado o mais próximo de onde as pessoas vivem e trabalham, e, assim, identificar, conhecer e intervir sobre as condições de saúde da população. Além disso, tem a possibilidade de identificar e intervir sobre as situações de trabalho domiciliar, que envolvem grande parcela dos trabalhadores, muitas vezes invisíveis às ações das políticas tradicionais de proteção da saúde no trabalho.

A estratégia de $A M$ às equipes da $A B$, ativada e incorporada pelo Cerest Regional de Betim, é considerada uma experiência de intervenção importante para o campo da ST. A adoção de práticas, como o atendimento individual compartilhado, a discussão de casos e temas técnicos específicos entre as equipes e o desenvolvimento conjunto de ações de vigilância epidemiológica propiciaram a incorporação de conceitos e práticas do campo da ST no cotidiano de trabalho das ESF.
Embora os profissionais da AB estejam absorvidos pelas atividades assistenciais e sobrecarregados pelo excesso de demanda, quando contam com retaguarda técnica especializada e pedagógica incorporam a perspectiva da ST em suas ações. Pode-se dizer que o AM produziu efeitos importantes, como: a ampliação do cuidado em ST; redução dos encaminhamentos aos Cerest, com aumento da resolutividade dos casos no âmbito da $A B$; maior articulação entre os profissionais da rede de $\mathrm{AB}$ e do Cerest; e maior qualificação dos encaminhamentos. Ademais, vale destacar que todos os profissionais das ESF afirmaram que o AM contribuiu para que compreendessem e incorporassem em suas atividades e abordagens o papel do trabalho enquanto determinante do processo saúde-doença.

Entre os desdobramentos do estudo destacam-se: a necessidade de ampliar a divulgação das experiências em curso e a formação dos apoiadores para operar o AM, garantindo, assim, a continuidade e se possível a ampliação dessa estratégia. Como resultado desse processo, espera-se mais qualidade da atenção à saúde dos trabalhadores no âmbito da $\mathrm{AB}$ e a inclusão de expressiva parcela de trabalhadores até o momento excluídos de um cuidado qualificado.

\section{Contribuições de autoria}

Lazarino MSA e Dias EC participaram do delineamento do projeto, coleta e análise dos dados, elaboração e revisão crítica do manuscrito. Silva TL participou da elaboração e revisão crítica do manuscrito. Todas as autoras participaram da aprovação final da versão publicada e assumem igual responsabilidade pelo seu conteúdo.

\section{Referências}

1. Dias EC, Hoefel MG. O desafio de implementar as ações de saúde do trabalhador no SUS: a estratégia da RENAST. Ciên Saúe Colet. 2005;10(4):817-27.

2. World Health Organization. Connecting health and labour. What role for occupational health in primary health care? Geneva: WHO; 2011 [citado em 19 maio 2019]. Disponível em: http://www. who.int/occupational_health/publications/hague_ executive_summary/en/index.html

3. Santibáñez Margüello M, Echabea EA, Medel GT, Montrull FB, Vioque Lópeza J. Percepción del personal médico de atención primaria de salud acerca de sus funciones, formación y conocimientos en materia de salud laboral. Atencion Primaria. 2008;40(1):7-12.

4. Dias EC, Lacerda e Silva T, Almeida MHC. Desafios para a construção cotidiana da Vigilância em Saúde Ambiental e em Saúde do Trabalhador na Atenção Primária à Saúde. Caderno de Saúde Coletiva. 2012;20(1):15-24.
5. Brasil. Ministério da Saúde. Portaria GM n ${ }^{\circ}$ 1679, de 19 de setembro de 2002. Dispõe sobre a estruturação da rede nacional de atenção integral à saúde do trabalhador no SUS e dá outras providências. Brasília: Ministério da Saúde; 2002.

6. Brasil. Ministério da Saúde. Portaria n ${ }^{0} 1.823 /$ GM, de 23 de agosto de 2012. Institui a Política Nacional de Saúde do Trabalhador e da Trabalhadora. Brasília: Ministério da Saúde; 2012.

7. Chiavegatto CV. Percepção dos profissionais de nível superior da atenção primária quanto ao desenvolvimento de ações de saúde do trabalhador no SUS em Minas Gerais. [dissertação]. Belo Horizonte: Universidade Federal de Minas Gerais; 2010.

8. Dias EC, Rigotto RM, Augusto LGS, Cancio J, Hoefel MGL. Saúde ambiental e saúde do trabalhador na atenção primária à saúde, no 
SUS: oportunidades e desafios. Ciên Saúde Colet. 2009;14(6):2061-70.

9. Campos GWS. Equipes de referência e apoio especializado matricial: uma proposta de reorganização do trabalho em saúde. Ciên Saúde Colet. 1999;4(2):393-404.

10. Campos GWS, Figueiredo MD, Pereira JN, Castro CP. A aplicação da metodologia paideia no apoio institucional, no apoio matricial e na clínica ampliada. Interface. 2014;18(Supl 1):983-95.

11. Pinto, AGA, Jorge MSB, Vasconcelos MGF, Sampaio JJC, Lima GP, Bastos VC, Sampaio HAC. Apoio matricial como dispositivo do cuidado em saúde mental na atenção primária: olhares múltiplos e dispositivos para resolubilidade. Ciên Saúde Colet. 2012;17(3):653-60.

12. Penido CMF. Análise da implicação de apoiadores e trabalhadores da estratégia de saúde da família no apoio matricial em saúde mental. [tese]. Belo Horizonte: Universidade Federal de Minas Gerais; 2012.

13. Madureira RMS, Bissoli SM. Apoio matricial em saúde do idoso: a experiência do município de Vitória-ES. In: Pinheiro R, Lopes TC, Silva FH, Silva Junior AG. (Orgs). Experienci(ações) e práticas de apoio e a integralidade no SUS: por uma estratégia de rede multicêntrica de pesquisa. Rio de Janeiro: Epesc/Abrasco; 2014. p. 135-47.

14. Fonseca Sobrinho D, Machado ATGM, Lima ÂMLD, Jorge AO, Reis CMR, Abreu DMX, Araújo LHL et al. Compreendendo o apoio matricial e o resultado da certificação de qualidade nas áreas de atenção à criança, mulher, diabetes/ hipertensão e saúde mental. Saúde Debate. 2014;38(spe):83-93.

15. Santos APL, Lacaz FAC. Apoio matricial em saúde do trabalhador: tecendo redes na atenção básica do SUS, o caso de Amparo/SP. Ciên Saúde Colet. 2012;17(5):1143-11.

16. Lakatos EM, Marconi MA. Fundamentos de metodologia científica. 7. ed. São Paulo: Atlas; 2010.

17. Minayo MCS. O desafio do conhecimento: pesquisa qualitativa em saúde. 11. ed. São Paulo: Hucitec; 2008.

18. Bardin L. Análise de conteúdo. [Rego L, Pinheiro A. trad.] Lisboa: Edições 70; 2006.

19. Campos GWS, Domitti AC. Apoio matricial e equipe de referência: uma metodologia para gestão do trabalho interdisciplinar em saúde. Caderno de Saúde Pública. 2007;23(2):399-407.

20. Dias EC, Lacerda-Silva T. Saúde do trabalhador na atenção primária à saúde: possibilidades, desafios e perspectivas. Rio de Janeiro: Coopmed; 2013.

21. Jorge MSB, Sousa FSP, Franco TB. Apoio matricial: dispositivo para resolução de casos clínicos de saúde mental na Atenção Primária à Saúde. Rev Bras Enferm [Internet]. 2013 [citado em 23 jan. 2014];66(5):738-44. Disponível em: http://www. redalyc.org/pdf/2670/267028883015.pdf

22. Oliveira GN. Devir apoiador: uma cartografia da função apoio. [tese]. Campinas: Universidade Estadual de Campinas; 2011. 